\title{
Quantum spiral bandwidth of entangled two-photon states
}

\author{
J. P. Torres, A. Alexandrescu and Lluis Torner \\ ICFO-Institut de Ciencies Fotoniques, \\ and Department of Signal Theory and Communications, \\ Universitat Politecnica de Catalunya, 08034 Barcelona, Spain
}

\begin{abstract}
We put forward the concept of quantum spiral bandwidth of the spatial mode function of the two-photon entangled state generated in spontaneous parametric down-conversion. We obtain the bandwidth using the eigenstates of the orbital angular momentum of the biphoton states, and reveal its dependence with the length of the down-converting crystals and waist of the pump beam. The connection between the quantum spiral bandwidth and the entropy of entanglement of the quantum state is discussed.

PACS numbers: 03.67.-a, 42.50.Dv
\end{abstract}


Entanglement, one of the most genuine features of quantum mechanics, is a basic ingredient in quantum cryptography, computing and teleportation [1, 2]. Spontaneous parametric down-conversion, namely the generation of two lower frequency photons when a strong pump field interacts with a nonlinear crystal, is a reliable source of entangled photons. The generated two-photon state is entangled in polarization [3], and most applications of parametric down-conversion in quantum systems make use of such spin-entanglement [4]-[ $]$. However, entanglement is also embedded in the spatial mode functions that describe the two-photon states 7]. Such spatial entanglement occurs in an infinite dimensional Hilbert space and is gaining increasing attention as a powerful way to encode and to exploit quantum information. 8, 9, 10, 11, 12, 13] For example, knowledge of the spatial structure acquired by the mode functions of entangled signal and idler photons forms the basis of multidimensional quantum imaging, and it can be used to increase the efficiency of multidimensional quantum communication protocols. The spatial structure of the photon states can be expressed by a mode decomposition of their mode function in an appropriate basis. The amount of entanglement of a quantum state is directly related to the width of such modal expansion, hereafter referred to as quantum spatial bandwidth. A two-photon state described by a single mode is a product state, while a quantum state described by an equi-distributed multimode expansion corresponds to a maximally entangled state. Therefore, a fundamental question that arises is how to act on the spatial quantum distribution of a given mode expansion, hence how the corresponding quantum information can be, e.g., concentrated.

It has been recently observed experimentally that the photon pairs generated in spontaneous down-conversion are entangled in orbital angular momentum (OAM) 14]. Such OAM entanglement allows the implementation of arbitrary $d$-dimensional quantum channels [15], thus it has been used to demonstrate violation of Bell inequalities with qutrits [16]. The corresponding mode functions are naturally expressed in terms of eigenstates of the paraxial OAM operator, whose spiral or winding topological structure can be resolved experimentally using combinations of holographic and filtering techniques. Therefore, such base provides a powerful tool to explore the concept of spatial multimode entanglement. In this Letter we put forward the full characterization of the entangled photon pairs in terms of eigenstates of the OAM operator and reveal how the corresponding quantum spiral bandwidth depends on the shape of the beam that pumps the down-converting crystal, and on the material properties and length on the crystal. We obtain in analytical form important similarity rules that 
hold for arbitrary input and crystal conditions.

Let a quadratic nonlinear crystal of length $L$ be illuminated by a quasi-monochromatic laser pump beam propagating in the $z$ direction. The spatial distribution of the pump beam writes $E_{p}(x, z, t)=E_{0}(x) \exp \left[i\left(\omega_{p} t-k_{p} z\right)\right]+c . c$, where $\omega_{p}$ is the angular frequency of the pump beam, $k_{p}$ is the wave number, $x$ is the position in the transverse plane and $E_{0}$ is the transverse spatial field profile at the input face of the crystal. The length of the crystal is assumed to be much smaller than the diffraction length $\left(L_{d}\right)$ of the pump beam $\left(L<<L_{d}\right)$, so that the spatial distribution of the pump beam in the transverse plane is independent of $z$. The signal and idler photons are assumed to be monochromatic, with $\omega_{p}=\omega_{s}+\omega_{i}$, where and $\omega_{s}$ and $\omega_{i}$ are the frequency of the signal and idler photons, respectively. This is justified by the use of narrow band interference filters in front of the detectors.

In the paraxial approximation, the spin and the OAM can be considered separately [17]. Under these conditions, photons described by a mode function that is a Laguerre-Gaussian $\left(\mathrm{LG}_{p}^{l}\right)$ mode are eigenstates of the OAM operator with eigenvalue $l \hbar$ 18]. The index $p$ is the number of non-axial radial nodes of the mode and the index $l$, referred to as the topological winding number, describes the helical structure of the wave front around a wave front singularity or dislocation. State vectors which are represented by a superposition of LG modes correspond to photons in a superposition state, with the weights of the quantum superposition dictated by the contribution of the $l$-th angular harmonics. When the pump beam is a $\mathrm{LG}_{p_{0}}^{l_{0}}$ mode, under conditions of collinear phase-matching, the two-photon state at the output of the nonlinear crystal can be written as a coherent superposition of eigenstates of the OAM operator [19] that are correlated in OAM, i.e $l_{1}+l_{2}=l_{0}$, where $l_{1}$ and $l_{2}$ refer to the OAM eigenvalues for the signal and idler photons. A photon state described by a LG mode can be written as

$$
\left|l p>=\int d q L G_{p}^{l}(q) a^{\dagger}(q)\right| 0>
$$

where the mode function in the spatial frequency domain writes

$$
\begin{aligned}
& L G_{p}^{l}(\rho, \varphi)=\left(\frac{w_{0}^{2} p !}{2 \pi(|l|+p) !}\right)^{1 / 2}\left(\frac{w_{0} \rho_{k}}{\sqrt{2}}\right)^{|l|} L_{p}^{|l|}\left(\frac{\rho_{k}^{2} w_{0}^{2}}{2}\right) \exp \left(-\frac{\rho_{k}^{2} w_{0}^{2}}{4}\right) \\
& \times \exp \left\{i l \varphi_{k}+i\left(p-\frac{|l|}{2}\right) \pi\right\},
\end{aligned}
$$

with $\rho_{k}$ and $\varphi_{k}$ being the modulus and phase, respectively, of the transverse coordinate $q$. The functions $L_{p}^{l}(\rho)$ are the associated Laguerre polynomials and $w_{0}$ is the beam width. 
The quantum state of the generated two-photon pair is given by [20]

$$
\left|\Psi>=\int d q_{s} d q_{i} \Phi\left(q_{s}, q_{i}\right) a_{s}^{\dagger}\left(q_{s}\right) a_{i}^{\dagger}\left(q_{i}\right)\right| 0,0>
$$

where $q_{s, i}$ are the transverse coordinates in the spatial frequency domain, $\mid 0,0>$ is the vacuum state, and $a_{s}^{\dagger}$ and $a_{i}^{\dagger}$ are creation operators for the signal and idler modes. Under conditions of collinear propagation of the pump, signal and idler photons, the mode function $\Phi\left(q_{s}, q_{i}\right)$ can be written in the paraxial approximation [21] as $\Phi\left(q_{s}, q_{i}\right)=E_{0}\left(q_{s}+q_{i}\right) W\left(q_{s}-q_{i}\right)$, where normalization of the state requires $\int d q_{s} d q_{i}\left|\Phi\left(q_{s}, q_{i}\right)\right|^{2}=1$. Here $E_{0}$ is the normalized field distribution of the pump beam in the spatial frequency domain, and the function $W$, which comes from the phase matching condition in the longitudinal direction, is given by

$$
W\left(q_{s}, q_{i}\right)=\sqrt{\frac{2 L}{\pi^{2} k_{p}}} \operatorname{sinc}\left(\frac{\left|q_{s}-q_{i}\right|^{2} L}{4 k_{p}}\right) \exp \left(-i \frac{\left|q_{s}-q_{i}\right|^{2} L}{4 k_{p}}\right) .
$$

One can decompose the quantum state $\mid \Psi>$ in the base of the eigenstates of the OAM operator, as

$$
\left|\Psi>=\sum_{l_{1}, p_{1}} \sum_{l_{2}, p_{2}} C_{p_{1}, p_{2}}^{l_{1}, l_{2}}\right| l_{1}, p_{1} ; l_{2}, p_{2}>,
$$

where $\left(l_{1}, p_{1}\right)$ correspond to the signal mode, $\left(l_{2}, p_{2}\right)$ correspond to the idler mode and the amplitude $C_{p_{1}, p_{2}}^{l_{1}, l_{2}}$ writes

$$
C_{p_{1}, p_{2}}^{l_{1}, l_{2}}=\int d q_{s} d q_{i} \Phi\left(q_{s}, q_{i}\right)\left[L G_{p_{1}}^{l_{1}}\left(q_{s}\right)\right]^{*}\left[L G_{p_{2}}^{l_{2}}\left(q_{i}\right)\right]^{*}
$$

The weights of the quantum superposition are given by $P_{p_{1}, p_{2}}^{l_{1}, l_{2}}=\left|C_{p_{1}, p_{2}}^{l_{1}, l_{2}}\right|^{2}$, which gives the value of the joint detection probability for finding one photon in the signal mode $\left(l_{1}, p_{1}\right)$ and one photon in the idler mode $\left(l_{2}, p_{2}\right)$. The two-photon state can be also characterized by the amplitude $A\left(x_{1}, z_{1}, t_{1} ; x_{2}, z_{2}, t_{2}\right)=<0,0\left|E_{1}^{+} E_{2}^{+}\right| \Psi>$, at positions $\left(x_{1}, z_{1}\right)$ and $\left(x_{2}, z_{2}\right)$ and times $t_{1}$ and $t_{2}$, where $E^{+}(x, z, t)$ is the electric field operator. If one makes use of the paraxial approximation for the electric field operator of a photon travelling in vacuum [20, 21], the amplitude can be written as

$$
A\left(x_{1}, z_{1}, t_{1} ; x_{2}, z_{2}, t_{2}\right)=\sum_{l_{1}, p_{1}} \sum_{l_{2}, p_{2}} C_{p_{1}, p_{2}}^{l_{1}, l_{2}} L G_{p_{1}}^{l_{1}}\left(x_{1}, z_{1}\right) L G_{p_{2}}^{l_{2}}\left(x_{2}, z_{2}\right) \exp \left(-i \omega\left(t_{1}+t_{2}\right)\right)
$$

Such amplitude might be employed, e.g., to draw analogies between the spatial structure of the two-photon quantum states and the properties of the corresponding incoherent classical radiation (see [12, 20]). 
The amplitudes $C_{p_{1}, p_{2}}^{l_{1}, l_{2}}$ depends on two normalised parameters: the normalised pump beam width $\bar{w}_{p}=w_{p} / \sqrt{\lambda_{p} L}$ and the normalised beam width of the LG modes, $\bar{w}_{0}=w_{0} / \sqrt{\lambda_{p} L}$, where $\lambda_{p}$ is the wavelength of the pump beam in vacuum. The assumption that the diffraction length $\left(L_{d}\right)$ of the pump beam is much smaller than the length of the crystal $\left(L<<L_{d}\right)$, requires the condition $\pi n_{p} \bar{w}_{p}>>1$ to be fulfilled, with $n_{p}$ being the refractive index of the pump beam inside the crystal. Here we always consider $\bar{w}_{p} \geq 1$. For a typical value of the pump wavelength $\lambda_{p}=0.4 \mu \mathrm{m}$, and a crystal length of $L=1 \mathrm{~mm}$, a pump beam width of $w_{p} \sim 20 \mu \mathrm{m}$ correspond to a normalized value of $\bar{w}_{p} \sim 1$.

In Figure 1 we plot the contribution to the mode decomposition of the quantum state $|\Psi\rangle$ of all the harmonics with the same value of the indexes $l_{1}$ and $l_{2}$, i.e. $P_{l_{1}, l_{2}}=\sum_{p_{1}, p_{2}=0}^{\infty} P_{p_{1}, p_{2}}^{l_{1}, l_{2}}$. In all cases cases the pump beam is a Gaussian mode. The distribution $P_{l_{1}, l_{2}}$ depends only on the normalised width $\bar{w}_{p}$. Thus, for a given material, the OAM distribution of the state $\mid \Psi>$ depends on the ratio $w_{p} / \sqrt{\lambda_{p} L}$, a result which gives an important scaling rule. In Figure 2(a) we show the dependence of $P_{l_{1}, l_{2}}$ on $\bar{w}_{p}$ for different values of $l_{1}$ and $l_{2}$, with $l_{1}+l_{2}=0$. One observes that the spiral bandwidth increases with $\bar{w}_{p}$, therefore it can be made larger by increasing the pump beam width or by decreasing the crystal length. Notice that by doing so the coupling efficiency in the detection state is also modified (see, e.g., [22]). Although the total contribution $P_{l_{1}, l_{2}}$ of modes with a given index $l_{1}$ and $l_{2}$ depends only on $\bar{w}_{p}$, the actual amplitude distribution $C_{p_{1}, p_{2}}^{l_{1}, l_{2}}$ depends also on $\bar{w}_{0}$. In Figure 2(b) we show the contributions of the modes with different index $p_{1}$ and $p_{2}$ for $\bar{w}_{p}=1$ and $l_{1}=l_{2}=0$, which is representative of a general case. We plot $C_{p_{\text {max }}}^{0,0}=\sum_{p_{1}, p_{2}=0}^{p_{\max }} P_{p_{1}, p_{2}}^{l_{1}, l_{2}}$ as a function of $p_{\max }$. The plot reveals that there is an optimal value of $\bar{w}_{0}$ for which the contribution of $P_{0,0}^{0,0}$ is maximum, and only a few modes with different index $p$ make significant contributions to the total weight. Away from the optimal $\bar{w}_{0}$ value, a large number of modes are required to represent the quantum state.

In most applications that make use of the OAM of the photons, one projects into a subspace of the complete Hilbert space that describes the mode function of the photon (e.g., 16]). This implies considering only a fraction of the mode space. Let us consider only modes with $p_{1}=p_{2}=0$, and thus thereafter we define $\left|l_{1}, l_{2}\right\rangle \equiv \mid l_{1}, p_{1}=0 ; l_{2}, p_{2}=0>$. In this subspace, the two-photon state can be written as

$$
\left|\Psi>=\sum_{l_{1}=-\infty}^{\infty} \sum_{l_{2}=-\infty}^{\infty} C_{0,0}^{l_{1}, l_{2}}\right| l_{1}, l_{2}>.
$$


Since the amplitude $C_{0,0}^{l_{1}, l_{2}}$ depends on both normalised parameters $\bar{w}_{p}$ and $\bar{w}_{0}$, the expansion given by Eq. (8) depends on the base of LG modes considered. In general, calculation of the amplitudes $C_{p_{1}, p_{2}}^{l_{1}, l_{2}}$ requires a 4 -dimensional integration in the spatial frequency domain. However, for a pump beam with a spatial field profile corresponding to a $\mathrm{LG}_{0}^{l_{0}}$ mode, we were able to obtain the value of the amplitude $C_{0,0}^{l_{1}, l_{2}}$ is analytic form. Namely,

$$
C_{0,0}^{l_{1}, l_{2}}=A_{0} \Gamma\left(\frac{\left|l_{0}\right|+\left|l_{1}\right|+\left|l_{2}\right|}{2}+1\right) \tan ^{-1} \frac{1}{i+\frac{w_{0}^{2} k_{p}}{2 L}}
$$

for $l_{1} \cdot l_{2}<0$, and

$$
\begin{aligned}
& C_{0,0}^{l_{1}, l_{2}}=A_{0} \sum_{n=0}^{\min \left(\left|l_{1}\right|,\left|l_{2}\right|\right)} \frac{\left|l_{1}\right| !\left|l_{2}\right| !}{\left(\left|l_{1}\right|-n\right) !\left(\left|l_{2}\right|-n\right) !(n !)^{2}}\left(\frac{w_{0}^{2}}{2 w_{p}^{2}+w_{0}^{2}}\right)^{-n} \frac{\Gamma\left(\frac{\left|l_{0}\right|+\left|l_{1}\right|+\left|l_{2}\right|-2 n}{2}+1\right)}{\left(1+i \frac{4 L}{w_{0}^{2} k_{p}}\right)^{\frac{n}{2}}} \\
& \times \Gamma(n) \sin \left(n \tan ^{-1} \frac{1}{i+\frac{w_{0}^{2} k_{p}}{2 L}}\right)
\end{aligned}
$$

for $l_{1} \cdot l_{2} \geq 0$. In these expressions, $A_{0}$ stands for

$$
A_{0}=(-1)^{\left|l_{0}\right|-\left|l_{1}\right|-\left|l_{2}\right|}\left(\frac{k_{p} w_{p}^{2}}{\pi L}\right)^{\frac{1}{2}}\left(\frac{w_{p}}{w_{0}}\right)^{\left|l_{p}\right|}\left(\frac{2 w_{0}^{2}}{2 w_{p}^{2}+w_{0}^{2}}\right)^{\frac{\left|l_{0}\right|+\left|l_{1}\right|+\left|l_{2}\right|}{2}+1} \frac{2^{\frac{\left|l_{0}\right|-\left|l_{1}\right|-\left|l_{2}\right|}{2}+1}}{\sqrt{\left|l_{0}\right| !\left|l_{1}\right| !\left|l_{2}\right| !}} .
$$

In both Eqs. (9) and (10), the relation $l_{0}=l_{1}+l_{2}$ holds. It is worth stressing that Eqs. (91)-(11) provide the analytical expression of the quantum state under general conditions in terms of the pump beam and the crystal properties. Moreover, this expansion corresponds to performing a Schmidt decomposition for continuos variables in this subspace, thus allowing to calculate the entropy of entanglement. 23]

In Figure 3, we plot the expansion in OAM eigenstates of the two-photon state for pump beams with $l_{0}=0,1,2$ and $\bar{w}_{p}=\bar{w}_{0}=1$. In the three cases represented, the subspace we are considering represents more than $40 \%$ of the corresponding complete Hilbert space. The spiral bandwidth of the expansion in terms of states $\mid l_{1}, l_{2}>$ increases with $l_{0}$. Equations (91) and (10) show that, for a given pump beam, the phase of the amplitude $C_{0,0}^{l_{1}, l_{2}}$ changes for each mode with $l_{1} \cdot l_{2}<0$, but it is the same for all modes with $l_{1} \cdot l_{2} \geq 0$.

The concept can be extended to the general case of a pump beam whose spatial field distribution is a coherent superposition of LG modes, so that $E_{0}(x)=\sum_{m} C_{m} L G_{0}^{m}(x)$, with $m=0,2,4 . . M$. The LG modes $L G_{0}^{m}(x)$ are the corresponding expression in the spatial domain of the LG modes given by Eq. (2). The energy flow $I$ of the pump beam can be written as [15] $I=2 \epsilon_{0} c n_{p} \sum_{m}^{M}\left|C_{m}\right|^{2}$. If we restrict to the subspace spanned by 
the states of the form $\mid l, l>$, one generates an entangled two-photon state of the form $\left|\Psi>\sim \sum_{n=0}^{N} \gamma_{n}\right| n, n>$. By making use of Eqs. (9) and (10), one obtains that the amplitudes $\gamma_{n}$ of the quantum state generated when the crystal is pumped by the superposition of LG modes are

$$
\gamma_{n}=\frac{\sqrt{(2 n) !}}{n !}\left[\frac{2 \bar{w}_{p}}{\bar{w}_{0}\left(2 \bar{w}_{p}^{2}+1\right)}\right]^{2 n+2} C_{2 n},
$$

This expression reveals that properly tailoring the spatial features of the beam that pumps the down-converting crystal allows generation of optimal quantum states for different quantum information protocols, such as the entanglement enhancement required for obtaining maximally entangled states. [24] One approach for the realization of entanglement concentration is to perform mode filtering operations on the two-photon state that is generated at the output of the crystal [25]. In contrast, Eq. (12) shows that the required quantum state could be obtained through appropriate engineering of the spatial properties of the pump beam [26]. It also shows that the phase of the state $\mid n, n>$ corresponds to the phase of the corresponding mode of the classical pump beam, $\arg \left\{C_{2 n}\right\}$, a result consistent with the experimental observation by $\mathrm{Ou}$ et. al., that the down-converted photons carry information about the phase of the pump beam [27].

In conclusion, we have obtained and analyzed the detailed quantum spatial structure of the two-photon entangled states generated in parametric down-conversion in terms of the eigenstates of the orbital angular momentum operator. We put forward the related concept of quantum spiral bandwidth, and showed its dependence on the pump beam and down-converting crystal. This allows, e.g., to define an effective finite Hilbert space where entanglement takes place.[28] Engineering such quantum spatial bandwidth should be a key tool to optimize multidimensional quantum information schemes, for example to increase the resolution of two-photon imaging using entangled photons (e.g., [29]) and to enhance the efficiency of relevant multidimensional quantum cryptography protocols (e.g., [30]).

This work was supported by the Generalitat de Catalunya and by the Spanish Government through grant BFM2002-2861. 
[1] M. A. Nielsen and I. L. Chaung, Quantum Computation and Quantum Information (Cambridge University Press, Cambridge, 2000).

[2] The Physics of Quantum Information, edited by D. Bouwmeester, A. Ekert, and A. Zeilinger (Springer, Berlin, 2000).

[3] P. G. Kwiat, K. Mattle, H. Weinfurter, A. Zeilinger, A. V. Sergienko, and Y. Shih, Phys. Rev. Lett. 75, 4337 (1995).

[4] D. Bouwmeester, J. W. Pan, K. Mattle, M. Eibl, H. Weinfurter, and A. Zeilinger, Nature (London) 390, 575 (1997).

[5] D. Bouwmeester, J. W. Pan, M. Daniell, H. Weinfurter, and A. Zeilinger, Phys. Rev. Lett. 82, 1345 (1999).

[6] T. Jennewein, C. Simon, G. Weihs, H. Weinfurter, and A. Zeilinger, Phys. Rev. Lett. 84, 4729 (2000).

[7] J. W. Simmons and M. J. Guttmann, States, Waves and Photons: A Modern Introduction to Light (Addison-Wesley, Reading, Mass., 1970).

[8] T. B. Pittman, D. V. Strekalov, D. N. Klyshko, M. H. Rubin, A. V. Sergienko, and Y. H. Shih, Phys. Rev. A 53, 2804 (1996).

[9] K. Banaszek, A. B. U'Rem, and I. A. Walmsley, Opt. Lett. 26, 1367 (2001).

[10] C. Simon and J. W. Pan, Phys. Rev. Lett. 89, 257901 (2002).

[11] P. Navez, E. Brambilla, A. Gatti, and L. A. Lugiato, Phys. Rev. A 65, 013813 (2002).

[12] A. Gatti, E. Brambilla, and L. A. Lugiato, Phys. Rev. Lett. 90, 133603 (2003).

[13] A. M. Dawes, M. Beck, and K. Banaszek, Phys. Rev. A 67, 032102 (2003).

[14] A. Mair, A. Vaziri, G. Weihs, and A. Zeilinger, Nature (London) 412, 313 (2001).

[15] G. Molina-Terriza, J. P. Torres, and L. Torner, Phys. Rev. Lett. 88, 013601 (2002).

[16] A. Vaziri, G. Weihs, and A. Zeilinger, Phys. Rev. Lett. 89, 240401 (2002).

[17] S. M. Barnett, J. Opt. B: Quantum Semiclass. Opt. 4, S7 (2002).

[18] L. Allen, M. J. Padgett, and M. Babiker, in Progress in Optics, edited by E. Wolf (Elsevier, Amsterdam, 1999), Vol. XXXIX, pp. 291-372.

[19] S. Franke-Arnold, S. M. Barnett, M. J. Padgett, and L. Allen, Phys. Rev. A 65, 033823 (2002).

[20] B. E. A. Saleh, A. F. Abouraddy, A. V. Sergienko, and M. C. Teich, Phys. Rev. A 62, 043816 
(2000).

[21] S. P. Walborn, A. N. de Oliveira, S. Padua, C. H. Monken, and B. E. A. Saleh, Phys. Rev. Lett. 90, 143601 (2003).

[22] F. A. Bovino, P. Varisco, A. M. Colla, G. Castagnoli, G. Di Giuseppe, and A. V. Sergienko, quant-ph/0300312s (2003).

[23] S. Parker, S. Bose and M. B. Plenio, Phys. Rev. A, 61, 032305 (2000)

[24] C. H. Bennett, H. J. Bernstein, S. Popescu, and B. Schumacher, Phys. Rev. A 53, 2046 (1996).

[25] A. Vaziri, J.W. Pen, T. Jennewein, G. Weihs, and A. Zeilinger, quant-ph/0303003 (2003).

[26] J. P. Torres, Y. Deyanova, L. Torner, and G. Molina-Terriza, Phys. Rev. A 67, 052313 (2003).

[27] Z. Y. Ou. L. J. Wang, X. Y. Zou, and L. Mandel, Phys. Rev. A 41, 566 (1990).

[28] C. K. Law, I. A. Walmsley, and J. H. Eberly, Phys. Rev. Lett. 84, 5304 (2000).

[29] A. F. Abouraddy, B. E. A. Saleh, A. V. Sergienko, and M. C. Teich, J. Opt. Soc. Am. B 19 1174 (2002).

[30] H. Bechmann-Pasquinucci and A. Peres, Phys. Rev. Lett. 85, 3313 (2000); C̆. Brukner, M. Żukowski, and A. Zeilinger, Phys. Rev. Lett. 89, 197901 (2002). 


\section{Figure captions}

Fig. 1: Mode distributions $P_{l_{1}, l_{2}}$ for different values of the parameter $\bar{w}_{p}$. (a) $\bar{w}_{p}=1$, (b) $\bar{w}_{p}=2.5$ and $(\mathrm{c}) \bar{w}_{p}=5$. The pump beam is a gaussian mode $\left(l_{p}=0\right)$, so that $l_{1}+l_{2}=0$. The $x$-axis represents the value of $l_{1}$.

Fig. 2: (a) Value of the weight $P_{l_{1}, l_{2}}$ for $\left(l_{1}, l_{2}\right)=(0,0),(1,-1)$ and $(2,-2)$, as a function of the normalized pump beam width $\bar{w}_{p}$. The labels show the value of $\left(l_{1}, l_{2}\right)$. The pump beam is a gaussian mode. (b) Weight $C_{p_{\max }}^{0,0}$ as a function of the index $p_{\max }$ for different values of $\bar{w}_{0}$, as shown in the labels. In all cases $\bar{w}_{p}=1$.

Fig. 3: Mode decomposition in the subspace $\mid l_{1}, p_{1}=0 ; l_{2}, p_{2}=0>$ for several pump beam. In (a), (c) and (e) we plot the weight $P_{0,0}^{l_{1}, l_{2}}$ of the mode distribution, and in (b), (d) and (f) we plot the phase $\arg \left\{C_{0,0}^{l_{1}, l_{2}}\right\}$ of each mode. (a), (b): $l_{0}=0 ;(\mathrm{c}),(\mathrm{d}): l_{0}=1$ and (e), (f): $l_{0}=2$. 
(a)

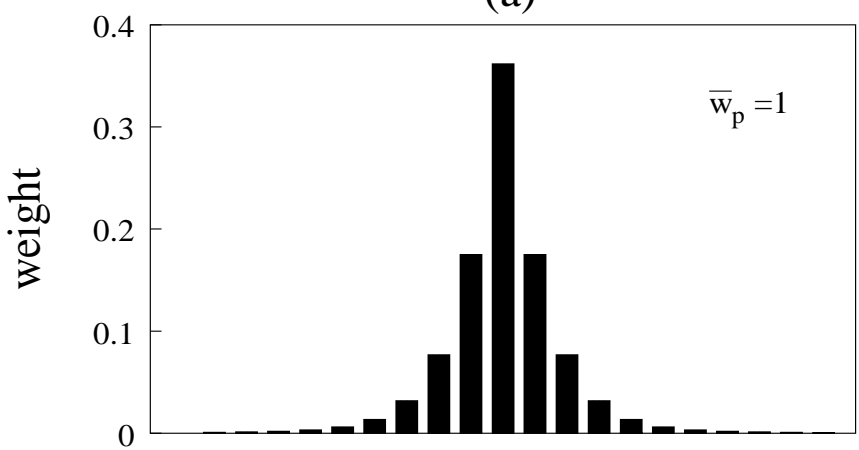

(b)
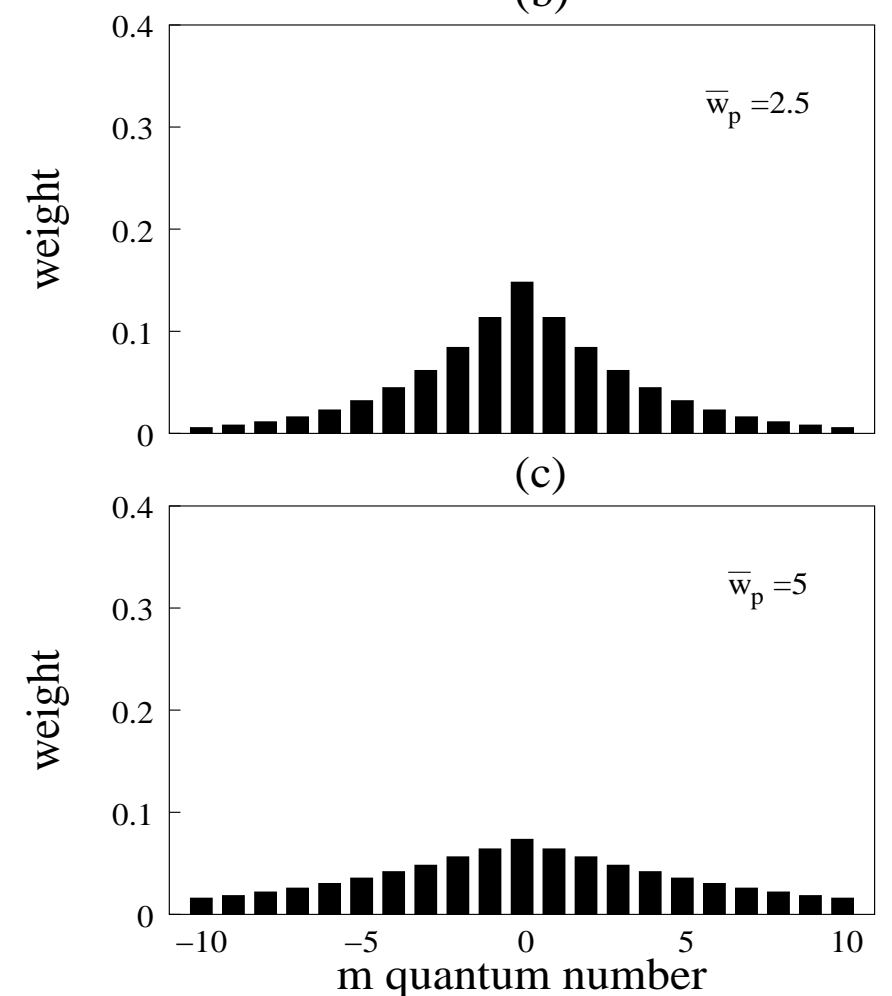
(a)

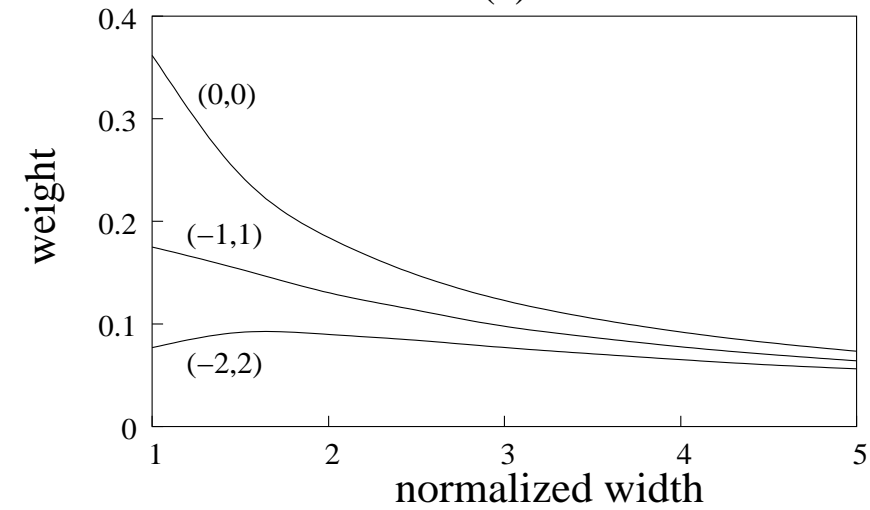

(b)

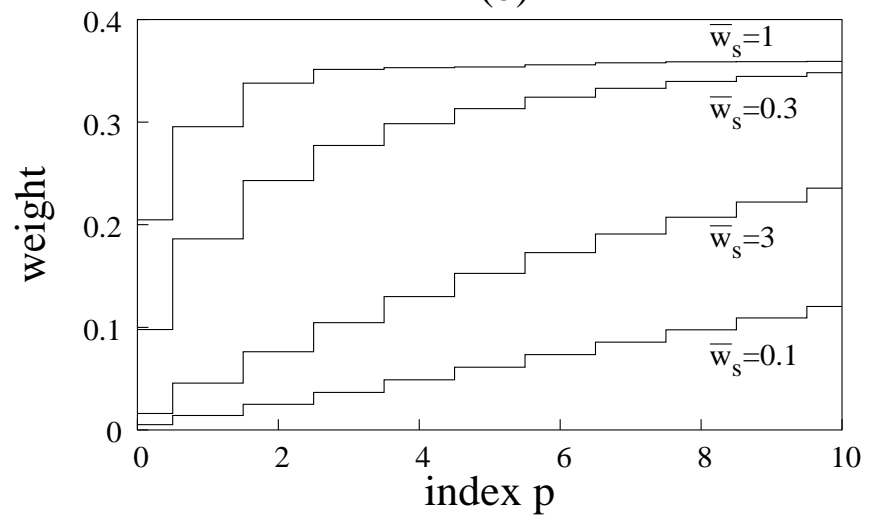


(a)

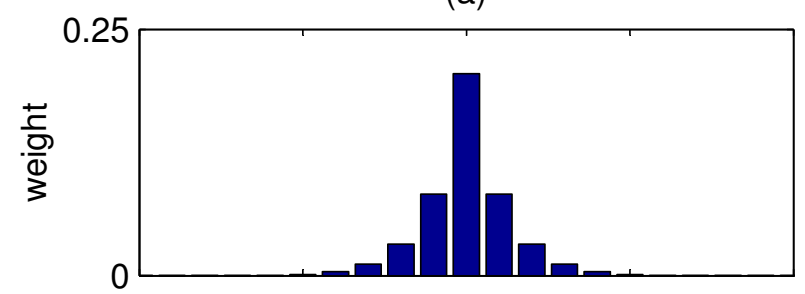

(c)

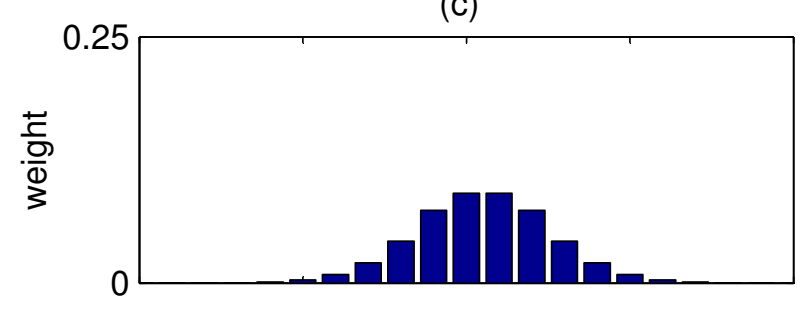

(e)

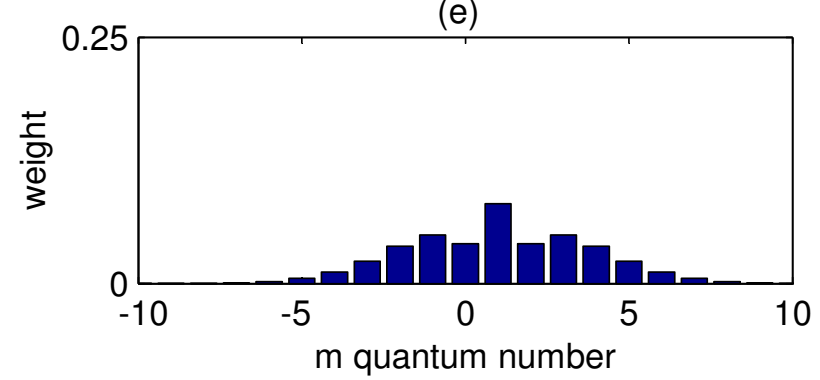

(b)

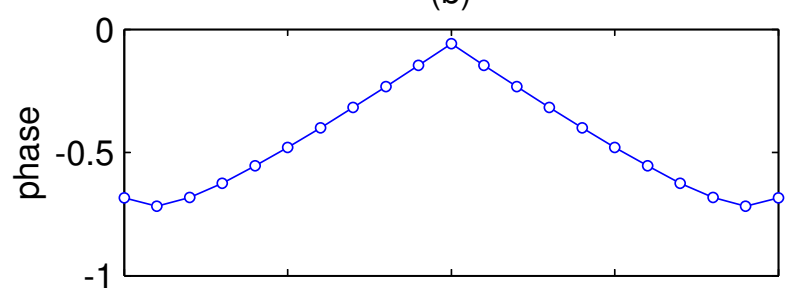

(d)
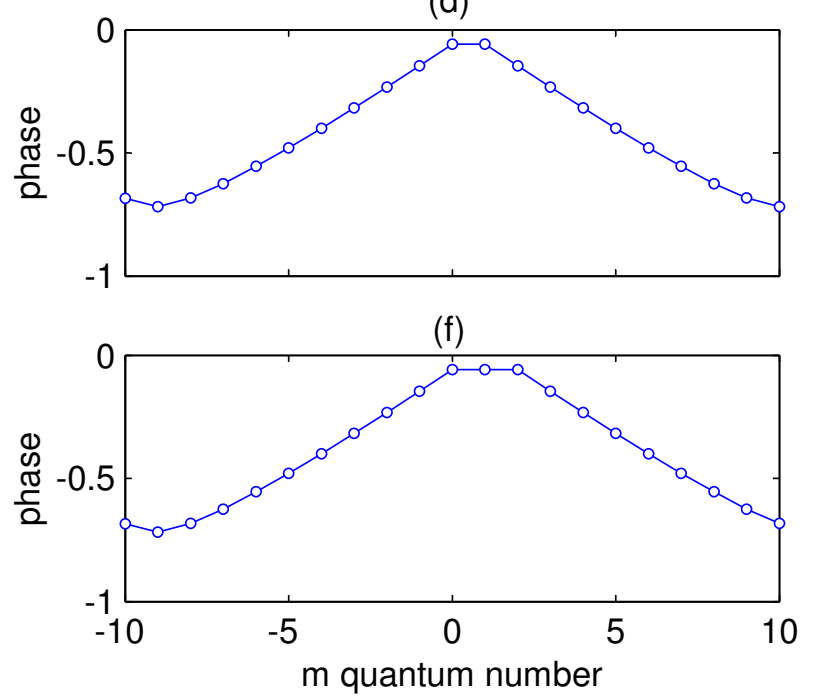